failure, size of VSD, posterior/inferior locations, and residual VSD. ${ }^{3}$ Management consists of VSD closure, coronary artery bypass grafting as needed, and aggressive postcardiotomy and end-organ support. Residual shunts are found in up to $40 \%$ of patients. Operative repair is associated with mortality greater than $60 \%,{ }^{4}$ which has prompted interest in percutaneous closure, but the role of these procedures is still undefined. ${ }^{5}$ In our case, the implantation of a biVAD served as a bridge to recovery. By minimizing associated postoperative end-organ damage with cardiac support, we allowed for physiologic recovery so the residual VSD could be successfully closed and the impact of the massive myocardial infarction and associated heart failure could be minimized. Limitations include the need for multiple procedures (ie, device weaning/explant), aggressive anticoagulation in a high-risk patient, and meticulous follow-up.

Right ventricular support is critical after the acute volume and pressure overload of a PI-VSD. Recovery may be unpredictable and prolonged, necessitating long-term biventricular support. Short-term univentricular options, such as intraaortic balloon pumps, extracorporeal membrane oxygenation, or left ventricular assist devices, are limited in right ventricle failure and residual shunting. A total artificial heart precludes native cardiac recovery and obligates transplantation; nevertheless, this may be an option with the appropriate resources and experience.

\section{Conclusion}

Biventricular mechanical support, as demonstrated in this complex case, should be considered a useful tool as a bridge to recovery in patients with PI-VSDs.

\section{References}

1. Cummings RG, Califf R, Jones RN, Reimer KA, King YH, Lowe JE. Correlates of survival in patients with postinfarction ventricular septal defect. Ann Thorac Surg. 1989;47:824-30 (update: 1997;63:1508-9).

2. Crenshaw BS, Granger CB, Birnbaum Y, et al. Risk factors, angiographic patterns and outcomes in patients with ventricular septal defect complicating acute myocardial infarction. GUSTO-1 (Global Utilization of Streptokinase and TPA for Occluded Coronary Arteries). Trial Investigators. Circulation. 2000;101:27-32.

3. Labrousse L, Choukroun E, Chevalier JM, et al. Surgery for postinfarction ventricular septal defect (VSD): risk factors for hospital death and long term results. Euro J Cardiothorac Surg. 2002;21: 725-31.

4. Jeppsson A, Liden H, Johnsson P, Hartford M, Radegran K. Surgical repair of post infarction ventricular septal defects: a national experience. Euro J Cardiothorac Surg. 2005;27:216-21.

5. Pienvichit P, Piemonte TC. Percutaneous closure of postmyocardial infarction ventricular septal defect with the CardioSEAL septal occluder implant. Catheter Cardiovasc Interv. 2001;54:490-4.

\title{
Atypical presentation of extralobar sequestration with absence of pericardium in an adult
}

\author{
Gourab Datta, MB, ChB, Jeymi Tambiah, MS, FRCS, Sheila Rankin, FRCR, Amanda Herbert, FRCPath, \\ and Loïc Lang-Lazdunski, MD, PhD, FRCS, London, United Kingdom
}

$\mathrm{E}$ xtralobar sequestration (ELS) is a rare congenital lesion of the lung. We report a case of ELS with concurrent absence of left pericardium presenting in an adult as an anterior mediastinal mass.

\section{Clinical Summary}

A 32-year-old man presented with a 3-week history of anterior chest discomfort. He denied any weight loss, night sweats, or fever. Clinical examination was unremarkable. Chest radiography and

From the Department of Thoracic Surgery, Guy's Hospital, London, United Kingdom.

Received for publication April 1, 2006; revisions received April 3, 2006; accepted for publication May 17, 2006.

Address for reprints: Loïc Lang-Lazdunski, MD, PhD, FRCS, Department of Thoracic Surgery, Guy's Hospital, St Thomas St, London SE1 9RT, United Kingdom (E-mail: loic.lang-lazdunski@gstt.nhs.uk).

J Thorac Cardiovasc Surg 2006;132:1239-40

$0022-5223 / \$ 32.00$

Copyright () 2006 by The American Association for Thoracic Surgery doi:10.1016/j.jtcvs.2006.05.054 computed tomography $(\mathrm{CT})$ revealed a cystic mass in the left anterior mediastinum $(80 \times 80 \times 70 \mathrm{~mm})$ in close proximity to the pulmonary trunk (Figure 1). Tumor markers, including $\alpha$-fetoprotein, $\beta$-human chorionic gonadotropin, and lactate dehydrogenase, were normal. A bronchogenic cyst or a thymic cyst was suspected. A CT-guided biopsy was performed but was inconclusive. The patient was referred to our department for biopsy or excision of the lesion.

We approached the mass through an anterior minithoracotomy via the left second intercostal space. An initial noteworthy finding was the absence of pericardium. A large cystic lesion with a smooth grayish surface was visualized, and amorphous brownish liquid was aspirated from it. Macroscopically, the lesion had the appearance of a bronchogenic cyst and was adherent to the mediastinum and pulmonary hilum. The mass was dissected from surrounding structures progressively by using diathermy and ligaclips, but extraction resulted in massive bleeding. The incision was enlarged, and a clamp was applied to the origin of the left pulmonary artery (LPA). The bleeding source was identified as a small vessel $(2 \mathrm{~mm})$ originating from the proximal LPA. It was controlled with a single purse-string suture. The mass was completely excised. The patient recovered uneventfully and was discharged on day 5 . 


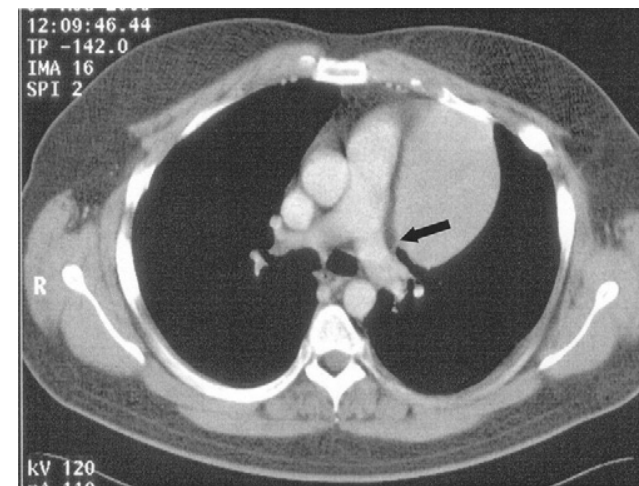

Figure 1. Chest computed tomographic scan showing a left-sided mediastinal mass in close proximity to the pulmonary artery. There is a small vessel containing the left pulmonary artery with the mass (arrow).

Retrospective analysis of the chest CT scan by a radiologist revealed a probable feeding vessel at the site of hemorrhage (Figure 1).

Histopathology demonstrated that the lesion had a wall made of bronchial tissue, with mucous glands and cartilage (Figure 2). The cyst was in lung parenchyma and invested by its own pleura. There were also small cysts and lung parenchyma (with airways containing calcified secretions) present within the tissue. There was no evidence of malignancy and no heterologous elements to suggest a dermoid cyst. The diagnosis was of an ELS.

\section{Discussion}

Pulmonary sequestration is a developmental defect consisting of variable amounts of nonfunctioning lung components that do not communicate with the tracheobronchial tree. It is distinguished from a bronchogenic cyst by the presence of lung parenchyma and also a dedicated blood supply. Pulmonary sequestrations have been classified into intralobar sequestration (ILS), surrounded by normal lung visceral pleura, and ELS, which has its own pleural investment. ILS is at least 3 times more common than ELS. ${ }^{1}$ ELS is 4 times more common in male patients than in female patients, usually occurs on the left (90\%), and usually occurs in the posterior cardiophrenic angle. Cases of ELS have also been reported in the mediastinum and diaphragm or rarely in a subdiaphragmatic position. Unlike ILS, ELS is frequently associated with other congenital anomalies, especially diaphragmatic hernias and foregut malformations. ELS usually presents in infants, although a few adult cases have been reported., ${ }^{2,3}$ There is only one previous report of ELS with absence of left pericardium in an adult. ${ }^{4}$

The arterial blood supply of ELS is predominantly from systemic arteries, usually the aorta, but arises from the pulmonary artery in less than $5 \%$ of cases. ${ }^{5}$ The venous drainage is through systemic veins, most often the azygos veins, hemiazygos veins, or inferior vena cava, although there is sometimes partial drainage through the pulmonary veins. ${ }^{4,5}$

In this case the mass appeared to have blood supply from the LPA. Venous drainage was not identified.

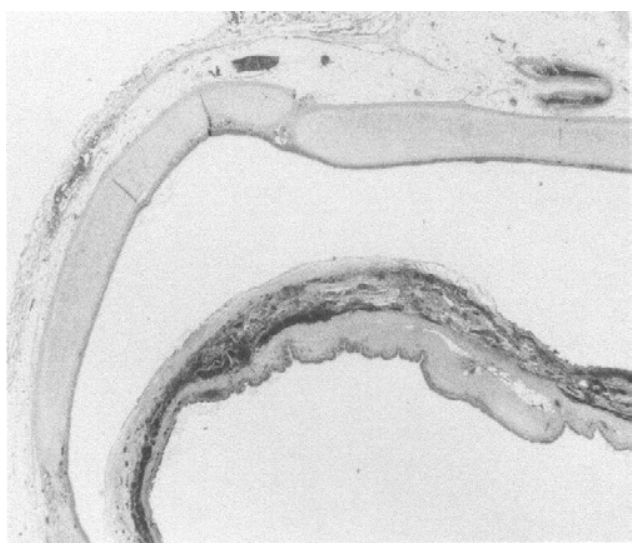

Figure 2. Microscopic appearance of the cystic lesion. (Hematoxylin and eosin stain, original magnification $10 \times$.) The cyst is lined by respiratory type epithelium and is seen in lung parenchyma invested by visceral pleura.

Surgical resection of pulmonary sequestration is the treatment of choice in symptomatic cases and provides tissue for diagnosis. In this case videothoracoscopic excision was not considered because of the large size of the mass and the absence of diagnosis. A 5-cm minithoracotomy was considered a good alternative and allowed easy dissection of the mass. The incision was enlarged into a $10-\mathrm{cm}$ anterior thoracotomy to control bleeding and provide good exposure of the pulmonary hilum.

Because bronchogenic cysts do not contain lung parenchyma in their walls, it was concluded that this mass was an ELS. This hypothesis was reinforced by the finding of a feeding vessel, which is uncommon in foregut cysts. The presence of a feeding vessel obviously confers a higher risk of bleeding during resection of these lesions compared with excision of foregut cysts. Despite careful review of chest CT scans, we did not establish the diagnosis preoperatively. ELS, and more generally pulmonary sequestration, should therefore be considered in the differential diagnosis of anterior mediastinal masses in adults, and the presence of feeding vessels should be actively sought preoperatively and during excision of such lesions.

\section{References}

1. Felker RE, Tonkin ILD. Imaging of pulmonary sequestration. AJR Am J Roentgenol. 1990;154:241-9.

2. Van Raemdonck D, De Boeck K, Devlieger H, Demedts M, Moerman P, Coosemans W, et al. Pulmonary sequestration: a comparison between pediatric and adult patients. Eur J Cardiothorac Surg. 2001;19:388-95.

3. Arslanian A, Leflour N, Hernigou A, Danel C, Riquet M. Complex extralobar sequestration in a 24-year-old woman. Ann Thorac Surg. 2003;76:2077-8.

4. Porte HL, Massouille DG, Lebuffe GR, Wurtz AJ. A unique congenital mediastinal malformation. Ann Thorac Surg. 2001;71:1703-4.

5. Amitai M, Konen E, Rozenman J, Gerniak A. Preoperative evaluation of pulmonary sequestration by helical CT angiography. AJR Am J Roentgenol. 1996;167:1069-70. 